\title{
Thermal Crosslinking of an Alkaline Anion Exchange Membrane Bearing Unsaturated Side Chains
}

\author{
Liang Wu ${ }^{1}$, Qi Pan ${ }^{1}$, John R. Varcoe ${ }^{2}$, Jin Ran ${ }^{1}$, Zhengjin Yang ${ }^{1}$, Tongwen Xu* \\ ${ }^{1}$ CAS Key Laboratory of Soft Matter Chemistry, Collaborative Innovation Center of Chemistry for \\ Energy Materials, University of Science and Technology of China, Hefei 230026, P.R. China. \\ ${ }^{2}$ Department of Chemistry, The University of Surrey, Guildford, Surrey GU2 7XH, United
}

Kingdom

*Corresponding author.Email: twxu@ustc.edu.cn.Tel: +86-551-63601587; Fax:

$+86-551-63602171$.

\begin{abstract}
We report a facile new route for the synthesis of self-crosslinked anion exchange membranes (AEM) without the need for any crosslinkers or catalysts. The soluble copolymers bearing flexible side chains, with alkene pendant groups, were synthesized via the Menshutkin reaction. The crosslinked derivatives were then prepared by the thermal crosslinking of the unsaturated side chains during the membrane formation process. ${ }^{1} \mathrm{H}$ NMR was used to determine the content of available alkene groups before crosslinking, while in situ FTIR spectroscopy was used to confirm successful thermal crosslinking. This approach, distinct from the use of classical post-crosslinking processes, installs ionic cross-links (bearing trimethyl quaternary ammonium hydroxide functionality) between the polymer chains. This is to mitigate against excessive water uptakes and dimensional swelling on hydration (extremely low swelling ratio of $2.5 \%$ in-plane and $1.2 \%$ through-plane $30{ }^{\circ} \mathrm{C}$ ), while retaining a high concentration of charge carriers (ion-exchange capacity) for target hydroxide conductivities. Additionally, the self-crosslinking strategy, and resulting dense crosslinked network, has the additional advantage of protecting the quaternary
\end{abstract}


ammonium groups from hydroxide ions attack. The strategy produced an AEM that yielded a peak power density of $42 \mathrm{~mW} \mathrm{~cm}^{-2}$ in a $\mathrm{H}_{2} / \mathrm{O}_{2}$ fuel cell at $60{ }^{\circ} \mathrm{C}$.

Keywords: anion exchange membrane; crosslinking; fuel cells; side chain; hydroxide conductivity

\section{Introduction}

Alkaline anion exchange membrane fuel cells (AAEMFCs) have received growing interest in recent years relative to acidic proton exchange membrane fuel cells (PEMFCs) stemming from the perceived improvements in the kinetics of the oxygen reduction at high $\mathrm{pH}$ and facilitated use of non-precious metal catalysts. ${ }^{1,2}$ As a critical component in AAEMFCs, an anion exchange membrane (AEM) often exhibit lower conductivities compared to the acidic analogues, proton exchange membranes (PEM), due to the intrinsically lower mobility of hydroxide ions compared to protons. ${ }^{3}$

In the past ten years, scientists have devoted increasing efforts to improve the hydroxide conductivities of AEMs via the introduction of highly basic ionic moieties or the tuning of the AEM's ordered micro-phase segregations. To increase the basicity of ionic moieties, phosphonium hydroxides ${ }^{4,5,6,7,8}$, guanidinium hydroxides ${ }^{9,10,11}$ (higher basicities compared to quaternized ammonium hydroxides) have been introduced by quaternization of halomethylated polymers using Menshutkin reaction. In general, the higher basicities of such ionic moieties results in an augmentation in both the number of dissociated hydroxides and the adsorbed water molecules, thereby facilitating hydroxide ion conduction. Although the development of recent novel AEMs represents a promising progress in the development of AEMs in recent years, they often continue to show much lower conductivities than perflorosulfonated PEMs, such as $\mathrm{Nafion}^{\circledR}\left(\mathrm{ca} .80 \mathrm{mS} \mathrm{cm}{ }^{-1}, 20^{\circ} \mathrm{C}\right)$, due to the inherently lower mobility of hydroxyl ions and the presence of less ordered self-assembly morphologies.

Recently, the unique molecular architecture of Nafion ${ }^{\circledR}$, which consists of hydrophobic polytetrafluoroethylene (PTFE) backbones and hydrophilic pendant 
perfluoroether side-chains terminated with sulfonic acid groups, has stimulated a whole new train of thought for membrane scientists, from the viewpoint of tuning the membrane morphologies of ionic side-chain-type AEMs. It is reported that ionic clusters, derived from aggregation of ionic side chains, can connect to each other to form continuous ionic transport channels ${ }^{12}$. Accordingly, a lot of AEMs bearing side chains, in which the pendant functional groups "hang on the top" 13 or interlink the side chains and main chains ${ }^{14,}{ }^{15}$, have been designed for morphology tuning purposes. To further improve the hydroxide conductivities, a larger number of ionic groups have been introduced onto the side-chains. Guiver et al. have reported AEMs containing pendant bis(phenyltrimethylammonium) groups ${ }^{16}$. Zhuang et al. have reported AEMs with side chain that contain double quaternize ammonium groups ${ }^{17}$. Our previous work has grafted quaternary ammonium-functionalized 4-vinylbenzyl chloride onto polymer backbones to prepare graft copolymer-type AEMs. The hydroxide conductivities varied with the lengths of the ionic grafts and reached a maximum of $45 \mathrm{mS} \mathrm{cm}^{-1}$ at $30{ }^{\circ} \mathrm{C} .{ }^{18,19}$ Increasing the ionic density of the side chains significantly enhances the polarity difference between the hydrophobic polymer backbones and the hydrophilic side chains, resulting in the formation of an ionic cluster morphology. However, materials with such high ionic contents generally suffer from undesirable, excessive water uptakes and dimensional swellings on hydration in water, unless new strategies are introduced to mitigate against this effect.

Covalent crosslinking has been considered as a promising technique to improve both the dimensional (swelling) and chemical stability of quaternary ammonium side chain-type AEMs. To date, typical in situ crosslinked AEMs have been developed via Friedel-Crafts electrophilic substitutions ${ }^{20,21}$ or olefin metathesis techniques $22,23,24$, which allow crosslinking during solution casting to yield AEMs with homogeneous morphologies. These in situ crosslinking strategies give the advantages of reduced water uptakes (swelling) and increased resistance to chemical degradation, without sacrificing conductivities relative to AEMs formed via post-crosslinking techniques involving additional crosslinkers. Moreover, crosslinks bearing ionic groups lead to increased ion incorporation, which in turn supports high conductivities. For example, 
Li and Hickner reported a crosslinked "comb-sharped" AEMs with conductivity of 40 $\mathrm{mS} \mathrm{cm} \mathrm{c}^{-1}$ and a low swelling ratio of $5.4 \%$ at $20{ }^{\circ} \mathrm{C}^{23}$.

Inspired by the in situ crosslinking strategy, we report a facile new route for self-crosslinked AEMs that does not require the use of crosslinkers or catalysts. Unsaturated side chains were attached onto aromatic backbones via the Menshutkin reaction and then in situ crosslinked during the membrane formation step via a thermal-only treatment. This approach incorporates crosslinks, bearing quaternary ammonium cations, between the polymer chains in order to mitigate against excessive water swelling and to enable the high ion contents required to provide favorable hydroxide conductivity. The effect of crosslinking on properties such as water swelling, ionic conductivity, membrane morphology, durability to alkali, and $\mathrm{H}_{2} / \mathrm{O}_{2}$ fuel cell performance is presented and discussed.

\section{Experimental}

\subsection{Materials}

Bromomethylated poly (2,6-dimethyl-1,4-phenylene oxide) (BPPO) was kindly supplied by Shandong Tianwei Membrane Technology Co. Ltd. (China). It was purified by dissolving into NMP, precipitating in methanol, and drying at $40{ }^{\circ} \mathrm{C} .{ }^{1} \mathrm{H}$ NMR indicates $57.8 \mathrm{~mol} \%$ bromobenzyl $\left(\mathrm{Ph}-\mathrm{CH}_{2} \mathrm{Br}\right)$ and $42.2 \mathrm{~mol} \%$ aryl bromide $(\mathrm{Ph}-\mathrm{Br})$ per repeat unit. 2-(Dimethylamino)ethyl methacrylate (DMAEMA) (purity = 99.5\%) was purchased from Energy Chemical (China), while $N$-methyl-2-pyrrolidone (NMP, AR grade), N,N-dimethylformamide (DMF, AR grade), chlorobenzene (CB, AR grade), dimethyl sulfoxide (DMSO, AR grade), trimethylamine (TMA, gas) were purchased from Shanghai-Sinopham Chemical Reagent Co. Ltd. (China). All reagents were used as received without further purification. Deionized water (resistivity $=18.2$ $\Omega \mathrm{cm}$ ?) was used in all experiments.

\subsection{Synthesis of soluble BPPO-DMAEMA copolymer}

Soluble BPPO-DMAEMA copolymers were synthesized using the Menshutkin reaction. Typically, BPPO (1.0 g) was dissolved in NMP $(15 \mathrm{~mL})$ at $20{ }^{\circ} \mathrm{C}$ in a dried 
$25 \mathrm{~mL}$ three-neck round bottom flask equipped with a magnetic stirring bar. After the complete the BPPO had dissolved completely, DMAEMA $(0.736 \mathrm{~mL})$ was added dropwise (BPPO:DMAEMA reaction ratio $=1: 1.5)$. The reaction mixture was stirred at $20{ }^{\circ} \mathrm{C}$ for $12 \mathrm{~h}$. The final BPPO-DMAEMA copolymers were obtained by depositing into anhydrous diethyl ether. Excess DMAEMA monomers were removed by repeatedly washing of the product copolymer with ethanol. The purified copolymers were dried under vacuum at $40{ }^{\circ} \mathrm{C}$ for $48 \mathrm{~h}$ before characterization using NMR. Additionally, to investigate the effect of reaction temperature, another three reactions were conducted with stirring over a range of elevated temperatures $\left(40{ }^{\circ} \mathrm{C}\right.$, $50{ }^{\circ} \mathrm{C}$ and $65^{\circ} \mathrm{C}$ ) in order to check for the gelation point.

\section{X. Synthesis of crosslinked AEMs}

To fabricate crosslinked membranes, the purified BPPO-DMAEMA copolymer (above) was dissolved in NMP to form a casting solution of $25 \mathrm{wt} \%$, which was then cast onto 4 separate glass plates. The cast films were then heated at $40{ }^{\circ} \mathrm{C}, 60{ }^{\circ} \mathrm{C}, 80$ ${ }^{\circ} \mathrm{C}$, and $100{ }^{\circ} \mathrm{C}$ (one temperature per glass plate) for the simultaneous removal of solvent and to perform crosslinking. Evaporation of NMP (with a high boiling point) take a long time but has the advantage of ensuring complete crosslinking. After this drying-curing process, flexible, transparent, and yellow-tinged AEMs were obtained: these are designated as BPPO-DMAEMA- $x(x=40,60,80$, and 100 - where $x$ represents the drying temperature used). All membranes were fully converted to the $\mathrm{Cl}^{-}$anion form via immersion in aqueous $\mathrm{NaCl}\left(1 \mathrm{~mol} \mathrm{~L} \mathrm{~L}^{-1}\right)$ solution at room temperature for $24 \mathrm{~h}$, followed by thorough washing with deionized water to traces of remove residual ions from the ion-exchange solution. The AEMs were stored in deionized water until required (see below).

\subsection{Characterizations}

The structures of the starting BPPO and the BPPO-DMAEMA copolymer were characterized using ${ }^{1} \mathrm{H}$ NMR Spectroscopy (DMX 300 NMR spectrometer with a ${ }^{1} \mathrm{H}$ resonance at $300 \mathrm{MHz}$ ). Chloroform-d (DCC13) and dimethylsulfoxide- $d_{6}$ $\left(\mathrm{CD}_{3} \mathrm{SOCD}_{3}\right)$ were used as solvents, respectively. In situ Fourier transform infrared 
(FTIR) analysis was performed at different temperatures using a Nicolet iS10 FTIR Spectrometer (Thermo Nicolet Corporation, USA) equipped with a DTGS detector and a HT-32 heated, demountable cell. A dry sample of BPPO-DMAEMA-40 was clipped within the transmission card holder and was mounted in the cell. A spectrum of the AEM was first collected at $30^{\circ} \mathrm{C}$ as a background (spectral resolution of 1 $\left.\mathrm{cm}^{-1}\right)$. Then, without moving the sample, spectra were recorded over a range of increasing temperatures from $30-100{ }^{\circ} \mathrm{C}$. Phase images of BPPO-DMAEMA-40, BPPO-DMAEMA-80, and BPPO-DMAEMA-100 were recorded using atomic force microscopy (veeco diInnova SPM) under a non-contact mode (lateral scan frequency of $0.1 \mathrm{~Hz})$.

The ion exchange capacities (IEC) of AEM samples were determine by calculating the amount of quaternary ammonium ions per gram dry membrane. Firstly, $\mathrm{Cl}^{-}$ions were exchanged by $\mathrm{SO}_{4}{ }^{2-}$ anions by immersing the AEM sample under study in aqueous $\mathrm{Na}_{2} \mathrm{SO}_{4}(0.5 \mathrm{~mol} \mathrm{~L}-1)$ for $8 \mathrm{~h}$. Then, the concentration of released $\mathrm{Cl}^{-}$ions was determined by titration using aqueous $\mathrm{AgNO}_{3}\left(0.1 \mathrm{~mol} \mathrm{~L}^{-1}\right)$ and $\mathrm{XXX}$ as the indicator. IEC values were calculated from the amount of consumed $\mathrm{AgNO}_{3}$ at the end point and data is expressed as mmol $\mathrm{g}^{-1}$ of dry membrane (in $\mathrm{Cl}^{-}$form).

Gravimetric water uptakes (WU) and the dimensional swelling ratio (in-plane and through-plane) were determined by measuring weight, length, and thickness changes (of the $\mathrm{OH}^{-}$form AEMs) before and after hydration. A sample of the membrane $(4 \mathrm{~cm}$ in length and $1 \mathrm{~cm}$ in width) was immersed in deionized water at a controlled temperature for $24 \mathrm{~h}$. The hydrated sample was then wiped with tissue paper (to remove excess surface water) and its mass and thickness were quickly measured. HOW WERE MEMBRANES DEHYDRATED? WU and dimensional swelling ratio were calculated as follows:

$$
\begin{aligned}
& \mathrm{WU}(\%)=100 \% \times\left(W_{\text {wet }}-W_{d r y}\right) / W_{d r y} \\
& \text { Through-plane dimensional swelling ratio }(\%)=100 \% \times\left(T_{\text {wet }}-T_{d r y}\right) / T_{d r y} \\
& \text { In- plane dimensional swelling ratio }(\%)=100 \% \times\left(L_{w e t}-L_{d r y}\right) / L_{d r y}
\end{aligned}
$$


where $W_{w e t}$ and $W_{d r y}$ were the mass of hydrated and dry AEM sample, $T_{\text {wet }}$ and $T_{d r y}$ were the thicknesses of the same sample when hydrated and dehydrated, respectively, and $l_{\text {wet }}$ and $l_{d r y}$ were the lengths of the sample when hydrated and dehydrated, respectively.

The $\mathrm{OH}^{-}$ion conductivity was measured following a commonly encountered, standard four-point probe technique ${ }^{25}$ using an Autolab PGSTAT 30 (Eco Chemie, Netherland) potentiostat alongside a Teflon conductivity cell. All of the AEM samples were immersed in aqueous $\mathrm{NaOH}\left(0.1 \mathrm{~mol} \mathrm{~L}^{-1}\right)$ at room temperature for $12 \mathrm{~h}$, followed by thorough washing and immersion in DI water for at least $12 \mathrm{~h}$ before testing. During the measurement, a membrane sample was clamped into the cell, which was then placed inside an oven with controlled temperature and humidity: measurements were immediately conducted in order to eliminate potential errors caused by the reaction of the $\mathrm{OH}^{-}$ions with dissolved carbon dioxide. The testing procedure was carried out in galvanostatic mode (monitored using a Bode plot) with a current amplitude of $0.1 \mathrm{~mA}$ and a frequencies range of $1 \mathrm{MHz}-50 \mathrm{~Hz}$. The ionic resistance of membrane was then obtained from a Nyquist plot. The $\mathrm{OH}^{-}$conductivity was calculated according to the following equation ${ }^{26}$ :

$$
\kappa=\frac{L}{R W d}
$$

where $R$ is the absolute ohmic resistance of the AEM sample, $L$ the distance between potential-sensing electrodes ( $=1 \mathrm{~cm}$ for the test cell used) and $W$ and $d$ are width $(=1$ $\mathrm{cm}$ ) and thickness of the membrane, respectively.

The alkali stabilities of the AEMs were tested by monitoring the change in $\mathrm{OH}^{-}$ conductivity after being immersed in alkali solution for controlled lengths of time. Typically, BPPO-DMAEMA-100 membrane samples of controlled shape $(1 \mathrm{~cm} \times 4$ $\mathrm{cm})$ were immersed in aqueous $\mathrm{NaOH}\left(1 \mathrm{~mol} \mathrm{~L}^{-1}\right)$ solutions at $60{ }^{\circ} \mathrm{C}$. Samples were removed, one by one, within a $400 \mathrm{~h}$ period, and each was washed thoroughly using deionized water (to remove the excess $\mathrm{NaOH}$ ) before being immersed in deionized water prior to the measurement of ionic conductivity. 
Fabrication of the membrane electrode assembly (MEA), and typical beginning-of-life $\mathrm{H}_{2} / \mathrm{O}_{2}$ fuel cell test procedures were as reported previously ${ }^{27}$, 28 . A mixture of the catalyst (20\%wt Pt/C, Johnson Matthey HISPEC 3000, supplied by Alfa Aesar) and poly(vinylbenzyl chloride) in ethyl acetate solvent was sonicated and then sprayed onto carbon paper gas diffusion electrode substrates, until Pt loadings of $0.4 \mathrm{mg} \mathrm{cm} \mathrm{cm}^{-2}$ (geometric) were achieved. The ink-treated electrodes were then immersed in $N, N, N^{\prime}, N^{\prime}$-tetramethylhexane-1,6-diamine (99\%, Acros Organics) overnight to form the crosslinked alkaline anion-exchange ionomer (designated SION1 alkaline ionomer), and then were thoroughly rinsed with deionized water.

The membrane electrode assembly (MEA) was prepared by sandwiching the $\mathrm{OH}^{-}$form BPPO-DMAEMA-100 AEM between two electrodes without hot-pressing. The beginning-of-life performances of the MEAs (active geometric area of $5.3 \mathrm{~cm}^{2}$ ) was recorded using a Scribner 850e fuel cell test station (Scribner Associates Inc., USA) without back pressurization of the gas supplies. Fuel cell test was conducted at $60{ }^{\circ} \mathrm{C}$, with $\mathrm{H}_{2}$ and $\mathrm{O}_{2}$ gases (anode and cathode, respectively) with flow rates of 600 $\mathrm{cm}^{3} \mathrm{~min}^{-1}$ and controlled at a relative humidity of $\mathrm{RH}=100 \%$ (calculated from the dew point temperatures of the gases and the cell temperature of $60{ }^{\circ} \mathrm{C}$ ).

\section{Results and discussion}

\subsection{Investigation of the crosslinking process}



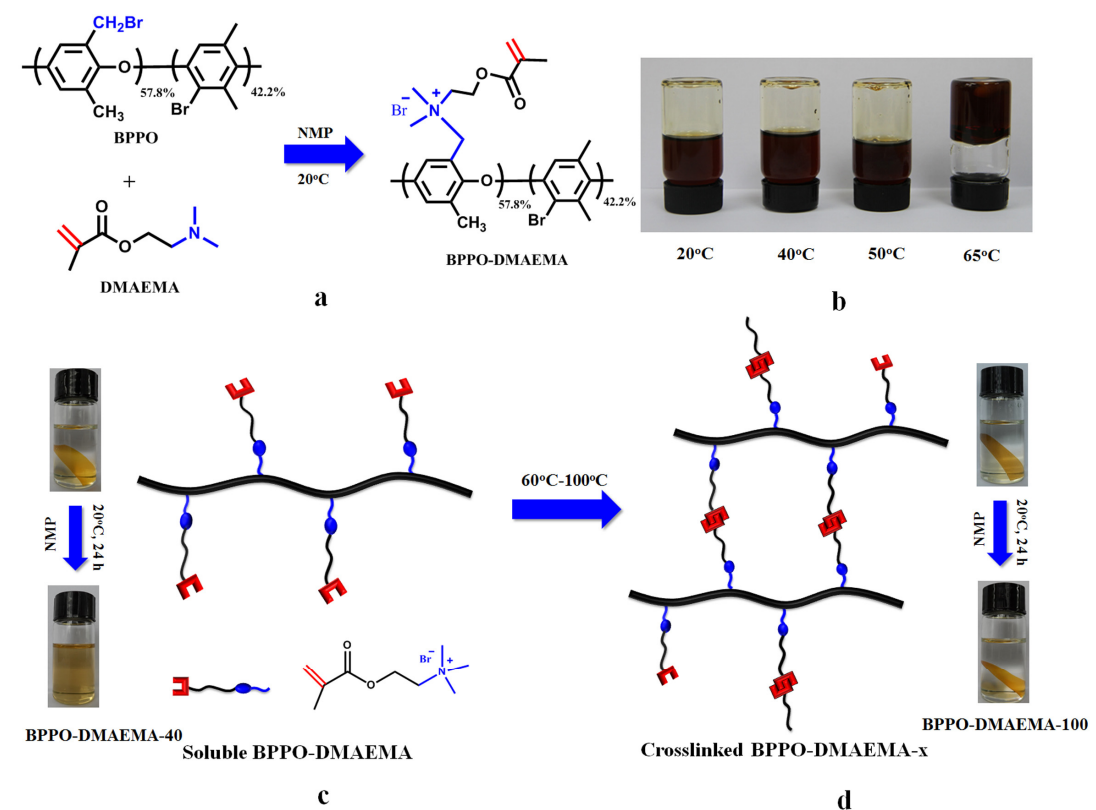

Fig. 1 The methodology used to produce the soluble intermediate BPPO-DMAEMA copolymer and the target crosslinked BPPO-DMAEMA- $x$ anion-exchange membranes (AEM): (a) Synthesis of the initial NMP soluble BPPO-DMAEMA copolymer; (b) The effect of the reaction on the production of BPPO-DMAEMA copolymer; (c) Schematic of the NMP soluble BPPO-DMAEMA- $x$ copolymers (drying $\mathrm{T}<50{ }^{\circ} \mathrm{C}$ ) and the NMP solubility of the $40{ }^{\circ} \mathrm{C}$ exemplar; (d) Schematic of the crosslinked BPPO-DMAEMA- $x$ AEMs (drying T $>60^{\circ} \mathrm{C}$ ) and the solubility of the $100{ }^{\circ} \mathrm{C}$ exemplar in NMP.

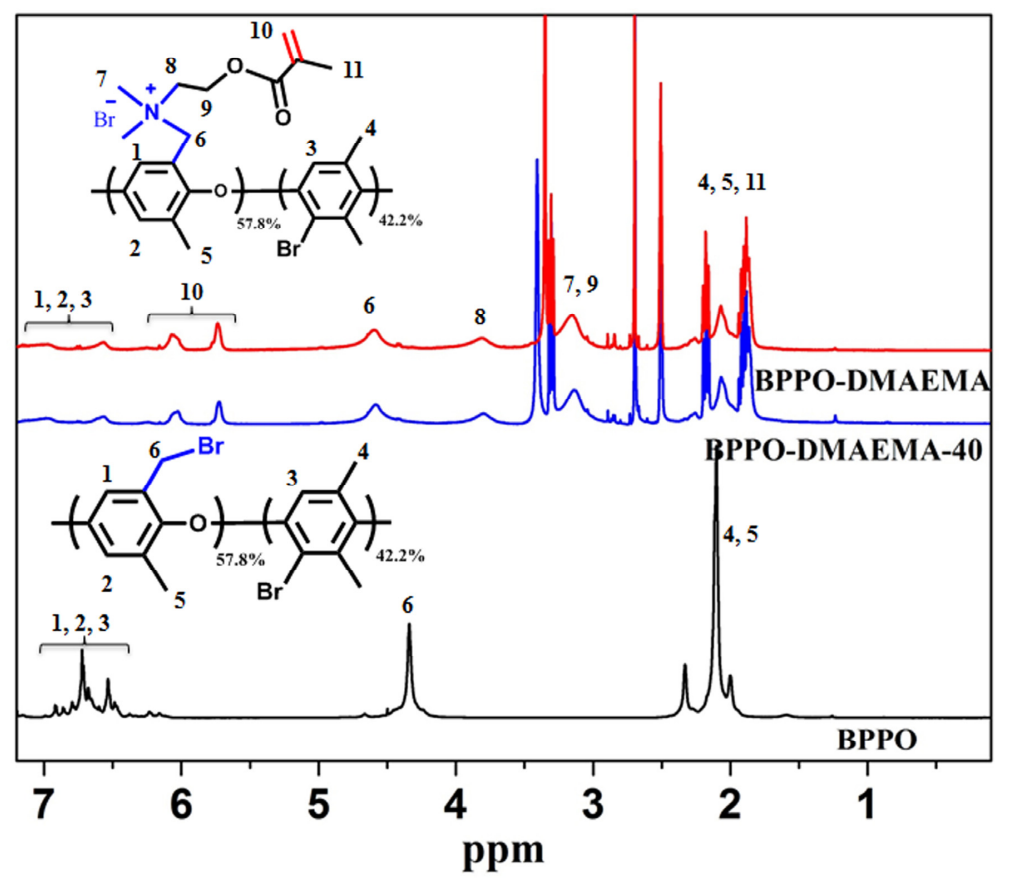

Fig. 2 The ${ }^{1} \mathrm{H}$ NMR spectra of BPPO, the NMP soluble BPPO-DMAEMA intermediate copolymer and the BPPO-DMAEMA-40 soluble copolymer. 
The soluble BPPO-DMAEMA copolymer and crosslinked BPPO-DMAEMA- $x$ AEM were prepared according to Fig. 1a. Copolymers bearing unsaturated side chains were synthesized using the Menshutkin reaction and a molar ratio of 1:1.5 between the BPPO bromomethyl groups and the DMAEMA tertiary amine groups. The ${ }^{1} \mathrm{H}$ NMR spectra of BPPO and BPPO-DMAEMA copolymer are presented in Fig. 2. The appearance of a peak at $3.2 \mathrm{ppm}$, which is ascribed to methyl or methylene groups on the QA groups, provide an initial indication of the success of this reaction. The peak due to the benzylic protons shifts from $4.3 \mathrm{ppm}$ to $4.5 \mathrm{ppm}$ due to the deshielding effects of the QA groups. The two protons signals expected for the pendant alkene functionality are observed at 5.6 and $5.8 \mathrm{ppm}$, indicating that alkene groups are not involved in any crosslinking while the reaction temperature is kept low $\left(20^{\circ} \mathrm{C}\right)$. However, the observations presented in Fig. $1 \mathrm{~b}$ suggests that the reaction is temperature dependent. When temperature is $<50{ }^{\circ} \mathrm{C}$, the reaction mixture remains transparent and homogeneous, but when a temperature of $65{ }^{\circ} \mathrm{C}$ is used the reaction leads to a gel. This provides evidence that the thermal initiated crosslinking, between pendant alkene groups, can occur at such temperatures with no need of catalyst.

The crosslinked AEMs were fabricated by casting a solution of BPPO-DMAEMA in NMP (25 wt $\%$ ) and slowly evaporating the (high boiling point) solvent at temperatures $>60{ }^{\circ} \mathrm{C}$. A schematic of the crosslinking process is illustrated in Fig. 1c and d. The inset to Fig. 1c (left) shows that BPPO-DMAEMA-40, dried at $40{ }^{\circ} \mathrm{C}$, immediately dissolves in NMP, suggesting that the pendant alkene groups had not crosslinked. The presence of alkene protons signals in the ${ }^{1} \mathrm{H}$ NMR spectrum of BPPO-DMAEMA-40 (Fig. 2) is consistent with this. In contrast, the BPPO-DMAEMA-40 membrane is insoluble in the NMP after further heating at 100 ${ }^{\circ} \mathrm{C}$ showing that the crosslinking strategy is viable. 


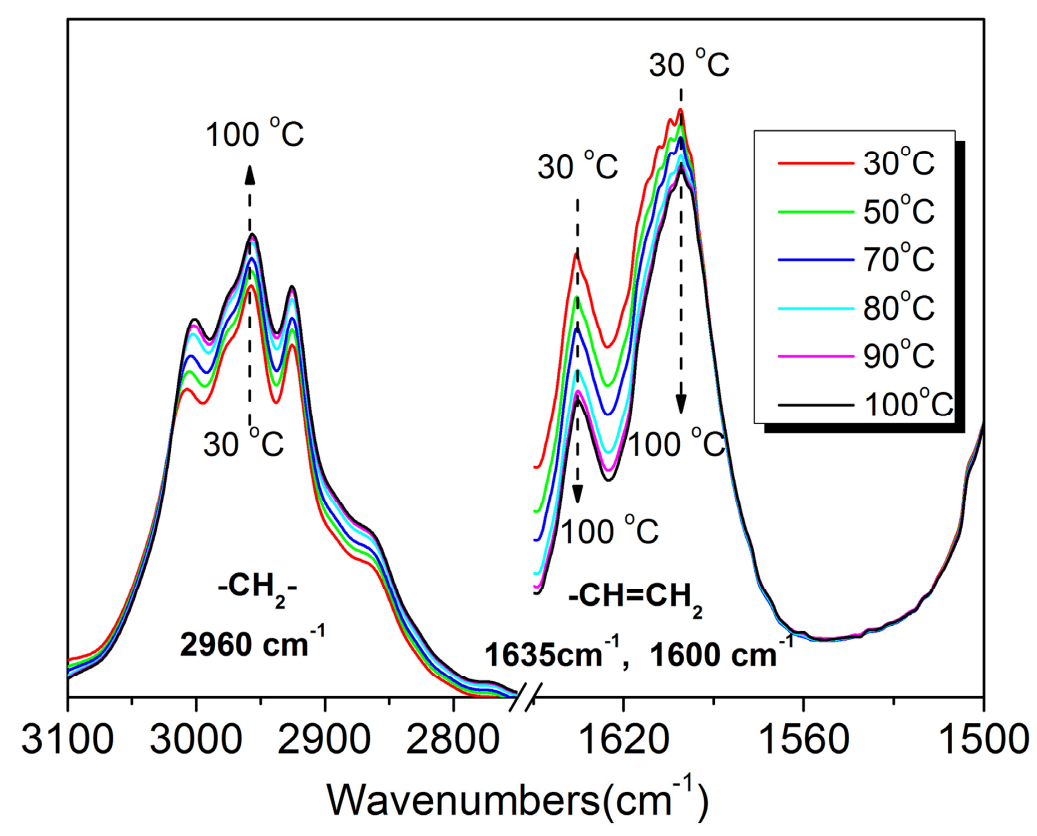

Fig. 3 FTIR spectra of BPPO-DMAEMA-40 AEM heated at different temperatures

In-situ FTIR analysis was employed to further investigate the temperature dependence of the self-crosslinking of the pendant alkene groups. The FTIR spectra of the initial un-crosslinked BPPO-DMA-40 membrane before and after increasing thermal treatment are presented in Fig. 3. The double peaks at 1630 and $1600 \mathrm{~cm}^{-1}$ are attributed to the vibrational modes of pendant alkene groups $\left(-\mathrm{CH}=\mathrm{CH}_{2}\right)$, while the multi-peak in the range of $2925-3075 \mathrm{~cm}^{-1}$ are attributed to the vibration of methylene $\left(-\mathrm{CH}_{2}-\right)$ groups. The intensity of the alkene peaks decreases on increasing temperature treatment $\left(30{ }^{\circ} \mathrm{C}\right.$ to $\left.100{ }^{\circ} \mathrm{C}\right)$. In contrast, the intensity of the methylene peaks increases on increased thermal treatment temperatures. The self-crosslinking process is clearly temperature dependent where more and more alkene groups react with each other, yielding crosslinked networks in the AEM, with the use of increased temperatures. 


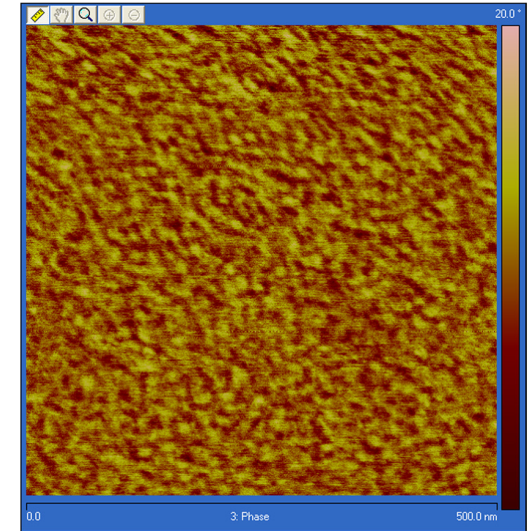

(a) BPPO-DMAEMA-40

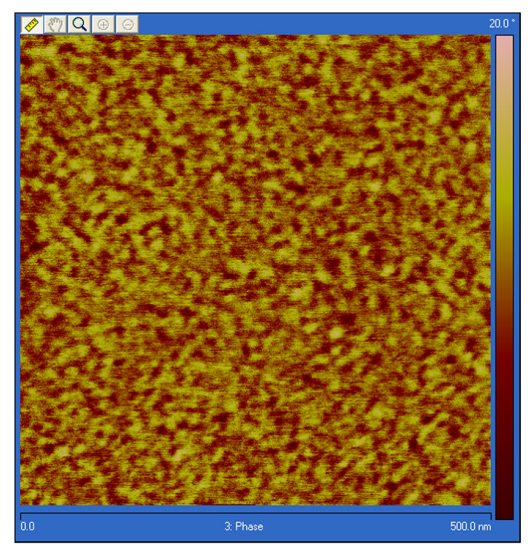

(c) BPPO-DMAEMA-100

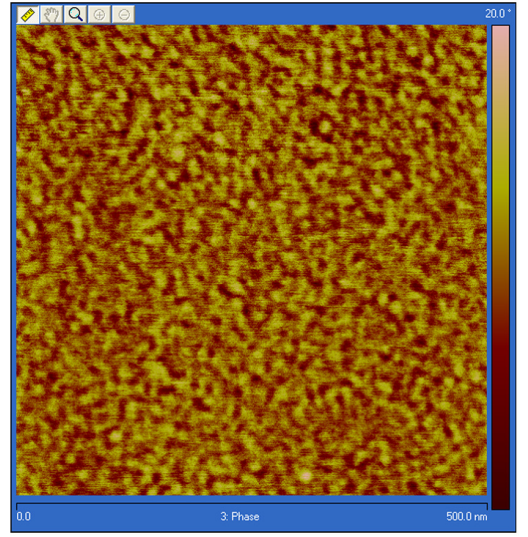

(b) BPPO-DMAEMA-80

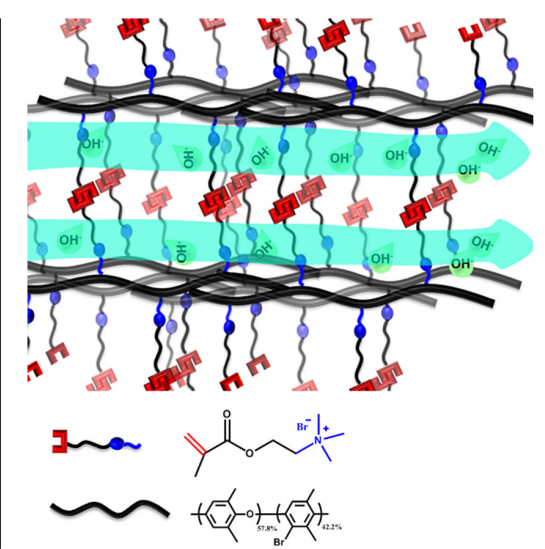

(d) Crosslinked network

Fig. 4 AFM phase images of: (a) BPPO-DMAEMA-40; (b) BPPO-DMAEMA-80; (c) BPPO-DMAEMA-100. (d) A simple schematic of the proposed crosslinked ionic network.

It should be noted that copolymers bearing flexible, functional side chains lead to the more facile formation of highly ordered morphologies with nano-phase separation (between the rigid main chains and flexible side chains). AFM analysis demonstrates that phase separation has occurred during the formation of the membranes in this study. The AFM phase image of BPPO-DMAEMA-40 (Fig. 4a) suggests that the quaternized unsaturated side chains form continuous hydrophilic channels (dark domains) in the hydrophobic main chain phase (light domains). Distinct nano-phase separation morphologies also were observed in the crosslinked AEMs (BPPO-DMAEMA-80 and BPPO-DMAEMA-100 in Fig. 4b and 4c), which suggests that the self-crosslinking process does not depress the degree of phase separation in the resulting AEMs. Considering that crosslinking happens between the side chains, it is possible to conclude that the crosslinked AEMs possess a much denser distribution of ion transport channels (illustrated in Fig. 4d), which looks promising considering 
our aim of producing AEMs with depressed water swelling (even at relative high temperatures). To see if this is the case, the effect of crosslinking on AEM properties such as IEC, water uptakes, dimensional swelling ratio, hydroxide conductivity, and alkaline stability was studied (see below).

\subsection{IEC, Water uptake and swelling ratio of crosslinked membranes}

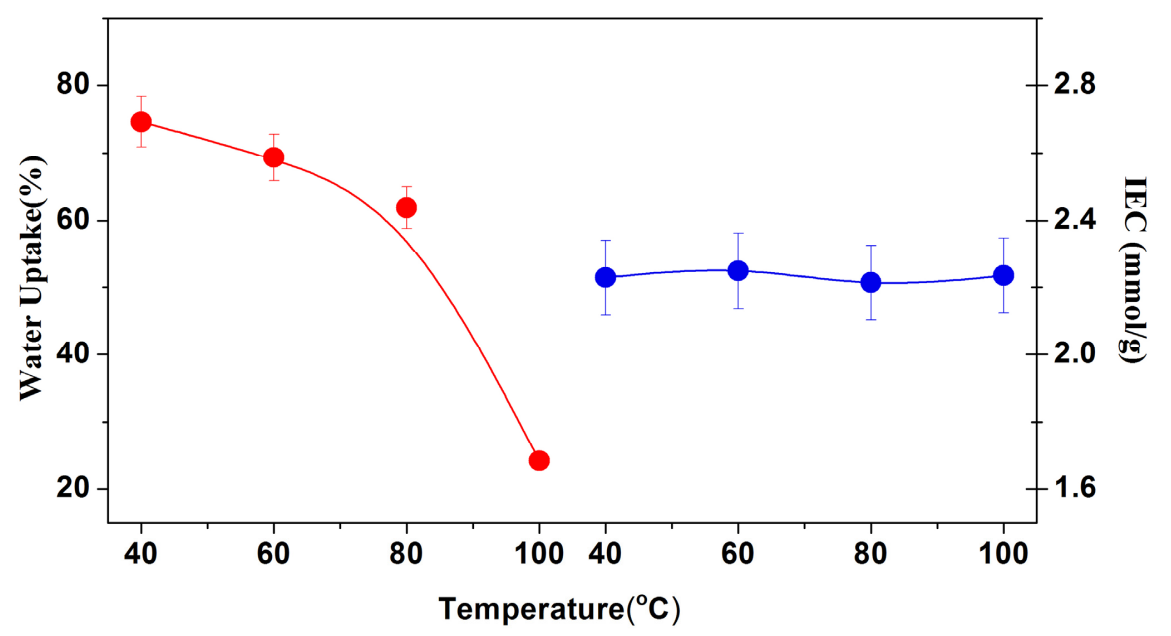

Fig. 5 Water uptake and IEC values of the AEMs crosslinked at the different temperatures.

Charged functional groups, which attract water molecules, play an important role in the construction of hydrophilic environments that support ion transport. Hence, increasing the ionic content (commonly measured as IEC) is a common strategy for the improvement of ion transport within an ion-conducting membrane. However, this strategy often leads to excessive membrane swelling on hydration (with high IECs). The aim of this study is to use self-crosslinking (of the ionic side chains) to construct dense hydrophilic networks to depress water swelling in the AEMs even if they possess high IECs. Fig. 5 presents the IECs and water uptakes of the AEMs formed using the various drying temperatures. The IECs remain invariant with drying temperature (at $c a 2.2-2.3 \mathrm{mmol} \mathrm{g}^{-1}$ ), whereas the water uptakes decrease from $75 \%$ to $24 \%$ on increasing drying temperature. According to the target reaction scheme presented in Fig. 1, no ionic groups or any atoms are sacrificed during crosslinking; the lack of mass change before and after crosslinking is consistent with the constant IEC values. In addition to water uptakes, excellent dimensional stabilities (preferably 
at high temperatures) is also required for real-world fuel cell operation. High dimensional changes in the membranes between dehydrated and hydrated states (either in the through-plane and even more significantly in-plane directions) will cause the separation of the electrodes from the membrane of the membrane electrode assembly (MEA). This will result in fuel leakage and voltage losses during fuel cell operation. Fig. 6 exhibits the dimensional swelling ratio of the BPPO-DMA-40 and BPPO-DMA-100 membranes in the in-plane and through-plane directions as a function of temperature. Specifically, the highly crosslinked BPPO-DMA-100 membrane possesses an extremely low in-plane SR of $2.5 \%$ and through-plane SR of $1.2 \%$ at $30{ }^{\circ} \mathrm{C}$, and retains excellent dimensional stabilities of $<15 \%$ swelling ratio at temperatures $<60{ }^{\circ} \mathrm{C}$. For un-crosslinked BPPO-DMA-40 membrane, it swells too excessively and falls into pieces when the temperature is $>40{ }^{\circ} \mathrm{C}$. This significant difference between these two exemplar membranes provides further evidence of the excellent control of swelling achieved with the self-crosslinked type.

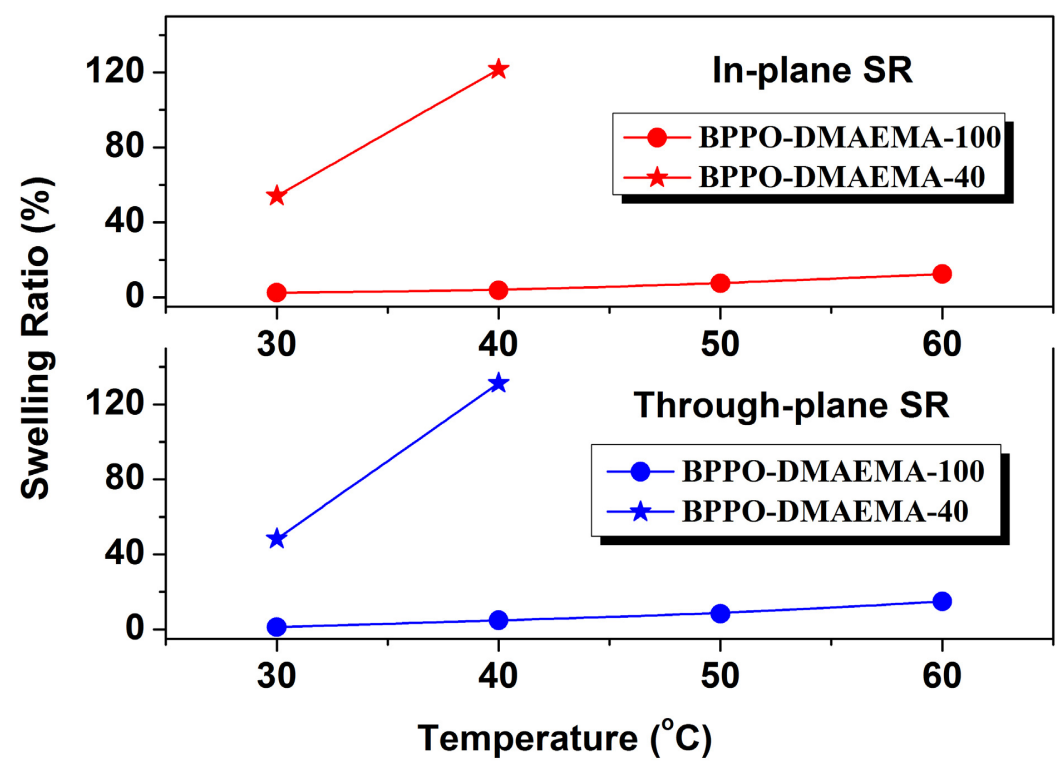

Fig. 6 In-plane and through-plane swelling ratio (Equations 2 and 3) of the BPPO-DMAEMA-40 and BPPO-DMAEMA-100 membranes.

\subsection{Fuel cell related performance}




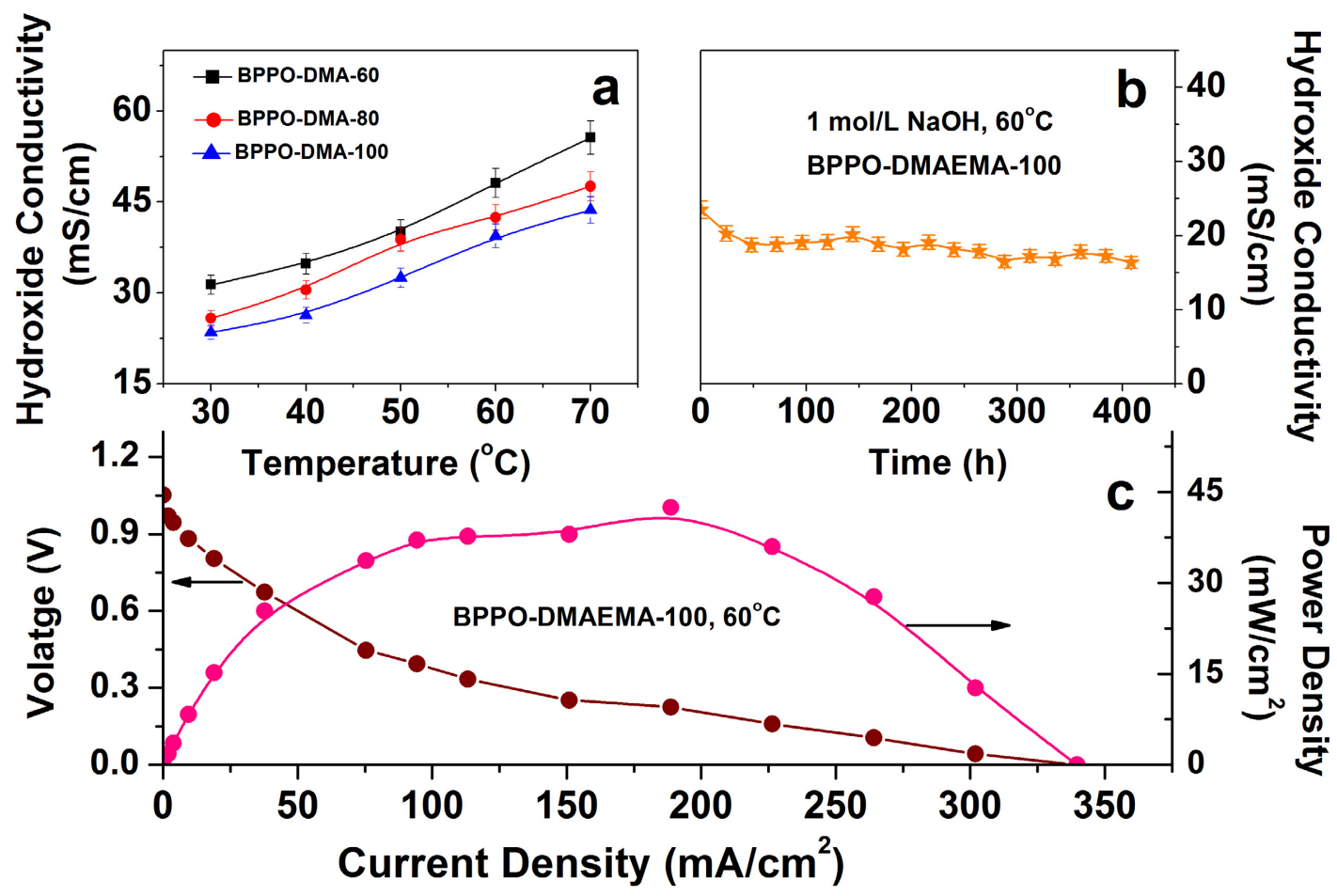

Fig. 7 The fuel cell-related performances of select membranes. (a) Hydroxide conductivity as a function of temperature; (b) Hydroxide conductivity of the BPPO-DMAEMA-100 AEM as a function of aging time [immersed in aqueous $\mathrm{NaOH}\left(1 \mathrm{~mol} \mathrm{~L}^{-1}\right)$ solutions at $60{ }^{\circ} \mathrm{C}$ ]; (c) Single cell beginning-of-life $\mathrm{H}_{2} / \mathrm{O}_{2}$ fuel cell performance of BPPO-DMAEMA-100 at $60{ }^{\circ} \mathrm{C}$ (fully hydrated gases with no back pressurization).

In addition to dimensional stability, favorable hydroxide conductivities and chemical stabilities are required for realistic fuel cell operation. Fig. 7 presents the hydroxide conductivities, an initial indication of alkaline stability, and an initial fuel cell performance of select crosslinked membranes. As expected, the hydroxide conductivities increase with temperature. However, the conductivity is dependent on the crosslinking temperature used in the synthesis process. For example, BPPO-DMA-60 possesses a conductivity of $32 \mathrm{mS} \mathrm{cm}^{-1}$ at $30{ }^{\circ} \mathrm{C}$, whereas BPPO-DMA-80 and BPPO-DMA-100 exhibit lower conductivities of $25 \mathrm{mS} \mathrm{cm}^{-1}$ and $21 \mathrm{mS} \mathrm{cm}^{-1}$, respectively. As shown in Fig. 5, all three membranes have the same IECs (i.e. the same amount of ionic groups per g of dry AEM) and vary in the degree of crosslinking. If uncrosslinked, it would be expected that they would adsorb the same amount of water molecules (as medium for hydroxide ions transport); the situation, however, changes when crosslinking occurs. During film formation at the lower drying temperatures, the flexible hydrophilic side chains twist to form 
hydrophilic clusters, which will swell until saturated by the adsorbed water molecules. When membrane formation occurs at the higher drying temperatures, the side chains twist and crosslink to each other to form much denser clusters, which will adsorb less water molecules due to the restraint of the denser crosslinked networks. A relatively high crosslinking degree appears to lead to a slight decrease in hydroxide conductivity. Moreover, the crosslinked membranes possess hydroxide conductivities of $21-35$ $\mathrm{mS} \mathrm{cm}{ }^{-1}$ at $30{ }^{\circ} \mathrm{C}$, which are comparable to other reported crosslinked $\mathrm{AEMs}^{22,23,24}$ and meets the basic requirement for application of the membranes in AAEMFCs $(>10$ $\mathrm{mS} \mathrm{cm}{ }^{-1}$ at $\left.25{ }^{\circ} \mathrm{C}\right){ }^{29}$. These results suggest that the thermal crosslinking strategy presented significantly mitigates against AEM swelling, whilst minimizing reductions in hydroxide conductivity. The BPPO-DMA-100 sample has the lowest swelling ratio but retains a favorable conductivity, suggesting its potential for application in AAEMFCs.

Alkaline stability is the biggest challenge with the development of AEMs (and ionomers) for AAEMFCs. For QA-type AEMs, degradation of the QAs by the the nucleophilic $\mathrm{OH}^{-}$ions is unavoidable (via $\beta$-hydrogen elimination and direct nucleophilic substitution at a $\alpha$-carbon ${ }^{2,30}$ ). In this study, the self-crosslinking strategy may have the advantage of protecting the QA groups from $\mathrm{OH}^{-}$attack. The BPPO-DMA-100 AEM remains intact, transparent, and flexible after immersion in 1 $\mathrm{M} \mathrm{NaOH}$ at $60{ }^{\circ} \mathrm{C}$ for $24 \mathrm{~d}$, while under comparable conditions typical benzyl trimethyl QA-based PPO membranes become very brittle due to severe degradation. Fig. $7 \mathrm{~b}$ shows the change of hydroxide conductivity of BPPO-DMAEMA-100 as a function of the alkali ageing time; the data shows a small but rapid initial decay followed by a levelling off of the conductivity loss. A drop in conductivity of $<20 \%$ of the initial conductivity over $48 \mathrm{~h}$ was observed at the ageing temperature of $60^{\circ} \mathrm{C}$. This gives a preliminary indication of the level of ex situ alkaline stability of BPPO-DMA-100. Longer term testing (along with more in depth studies including in situ stabilities) is, however, required as future work to further study the long term stabilities. 
Fig. 6c shows the initial $\mathrm{H}_{2} / \mathrm{O}_{2}$ fuel cell performance of BPPO-DMA-100 at $60{ }^{\circ} \mathrm{C}$. A peak power density of $42.5 \mathrm{~mW} \mathrm{~cm}^{-2}$ at a current density of about $200 \mathrm{~mA} \mathrm{~cm}^{-2}(\mathrm{X}$ V) was achieved. The performance may be enhanced if there is an improvement in the chemical compatibility between alkaline ionomer in the electrode used (non-PPO polystyrenic system) and the crosslinked AEM (PPO-based); the ionomer, which was prepared by treatment of poly(vinylbenzyl chloride) with $N, N, N^{\prime}, N$ '-tetramethylhexane-1,6-diamine ${ }^{26,27}$, shows a poor chemical compatibility with the crosslinked AEM leading to the high contact resistances and poor MEA lamination observed. Further effort is to be made in the future to improve the fuel cell performance by developing suitable alkaline ionomers and via the optimization of the testing conditions.

\section{Conclusion}

In summary, self-crosslinked AEMs were prepared by a novel, facile route without the needs of additional crosslinkers or catalysts. NMP soluble copolymers, bearing flexible side chains with alkene pendant groups, were initially synthesized and these were then thermally crosslinked during the membrane formation step. The in situ crosslinking significantly mitigates against water swelling (extremely low swelling ratios of $2.5 \%$ and $1.2 \%$ [in-plane and through-plane, respectively] at $30{ }^{\circ} \mathrm{C}$ were achieved with the $100{ }^{\circ} \mathrm{C}$ dried membrane) without sacrificing the amount of charge carriers (ion-exchange capacity) or the ionic hydroxide conductivities. Additionally, initial indications suggest that the self-crosslinking strategy can lead to reasonable alkali stability (via protection, from $\mathrm{OH}^{-}$attack, of the quaternary ammonium groups via the presence of the dense crosslinked network morphology. The AEM yielded an initial $\mathrm{H}_{2} / \mathrm{O}_{2}$ fuel cell performance of $42 \mathrm{~mW} \mathrm{~cm}^{-2}$.

\section{Acknowledgements}

We thank the financial supports from the National Basic Research Program of China (No. 2012CB932802), the National Natural Science Foundation of China (nos. 21376232, 51273185), National High Technology Research and Development 
Program 863 (2012AA03A608). John Varcoe an EPSRC Fellow funded on grant $\mathrm{EP} / \mathrm{I} 004882 / 1$.

References [ADD JVarcoe, TWXu big review paper? J. R. Varcoe, P. Atanassov, D.

R. Dekel, A. M. Herring, M. A. Hickner, P. A. Kohl, A. R. Kucernak, W. E. Mustain,

K. Nijmeijer, K. Scott, T. Xu, L. Zhuang, "Anion-exchange membranes in electrochemical energy systems", Energy Environ. Sci., 7, 3135 (2014)

[1] J. S. Spendelow, A. Wieckowski, Phys. Chem. Chem. Phys., 2007, 9, 2654-2675.

[2] J. R Varcoe, R. C. T Slade, Prospects for alkaline anion-exchange membranes in low temperature fuel cells. Fuel Cells 2005, 5,187-200.

[3] M. R. Hibbs, M. A. Hickner, T. M. Alam, S. K. McIntyre, C. H. Fujimoto, C. J. Cornelius, Transport Properties of Hydroxide and Proton Conducting Membranes, Chem. Mater. 2008, 20, 2566-2573.

[4] C.G. Arges, J. Parrondo, G. Johnson, A. Nadhan, V. Ramani, Assessing the influence of different cation chemistries on ionic conductivity and alkaline stability of anion exchange membranes. J Mater Chem 2012, 22, 3733-3744.

[5] K. K. Stokes, J. A. Orlicki, F. L. Beyer, RAFT polymerization and thermal behavior of trimethylphosphonium polystyrenes for anion exchange membranes. Polym Chem 2011, 2, 80-82.

[6] S. Gu, R. Cai, Y. S. Yan, Self-crosslinking for dimensionally stable and solvent-resistant quaternary phosphonium based hydroxide exchange membranes. Chem Commun 2011, 47, 2856-2858.

[7] S. Gu, R. Cai, T. Luo, Z. W. Chen, M. W. Sun, Y. Liu, G. H. He, Y. S. Yan, A Soluble and Highly Conductive Ionomer for High-Performance Hydroxide Exchange Membrane Fuel Cells. Angew Chem Int Ed 2009, 48, 6499-6502.

[8] K.J.T. Noonan, K.M. Hugar, H.A. Kostalik, E.B. Lobkovsky, H.D. Abruña, G.W. Coates. Phosphonium-functionalized polyethylene: a new class of base-stable alkaline anion exchange membranes. J Am Chem Soc 2012, 134,18161-18164.

[9] D.S. Kim, A. Labouriau, M.D. Guiver, Y.S. Kim, Guanidinium-functionalized anion exchange polymer electrolytes via activated fluorophenyl-amine reaction. Chem Mater 2011, 23, 3795-3797.

[10] J.H. Wang, S.H. Li, S.B. Zhang, Novel Hydroxide-Conducting Polyelectrolyte Composed of an Poly(arylene ether sulfone) Containing Pendant Quaternary Guanidinium Groups for Alkaline Fuel Cell Applications, Macromolecules 2010, 43, 3890-3896.

[11]X.C. Lin, L. Wu, Y.B. Liu, A.L. Ong, S.D. Poynton, J.R. Varcoe, T.W. $\mathrm{Xu}$, Alkali resistant and conductive guanidinium-based anion-exchange membranes for alkaline polymer electrolyte fuel cells, J Power Sources 2012, 217, 373-380.

[12]K.A. Mauritz, R.B. Moore, State of Understanding of Nafion. Chem. Rev.2004;104: 4535-4586. 
[13]N.W. Li, M. D. Guiver, W.H. Binder, Towards High Conductivity in Anion-Exchange Membranes for Alkaline Fuel Cells, ChemSusChem 2013, 6, $1376-1383$.

[14]N.W. Li, T.Z. Yan, Z. Li, T. Thurn-Albrecht, W. H. Binder, Comb-shaped polymers to enhance hydroxide transport in anion exchange membranes Energy Environ. Sci., 2012, 5, 7888-7892

[15]N.W. Li, Y.J. Leng, M. A. Hickner, C.Y. Wang, Highly Stable, Anion Conductive, Comb-Shaped Copolymers for Alkaline Fuel Cells, J. Am. Chem. Soc. 2013, 135, 10124-10133.

[16] S.Y. Lee, N.R. Kang, D.W. Shin, C.H. Lee, K.S. Lee, M.D. Guiver, N.W. Li, Y.M. Lee, Morphological transformation during cross-linking of a highly sulfonated poly(phenylene sulfide nitrile) random copolymer, Energy Environ Sci 2012, 5, 9795-9802.

[17]J. Pan, Y. Li, J.J. Han, G.W. Li, L.S. Tan, C. Chen, J.T. Lu, Lin Zhuang, A Strategy for Disentangling the Conductivity-Stability Dilemma in Alkaline Polymer Electrolytes, Energy Environ. Sci., 2013, 6, 2912-2915.

[18] J. Ran, L. Wu, B. Wei, Y.Y. Chen and T.W. Xu, Simultaneous Enhancements of Conductivity and stability for Anion Exchange Membranes (AEMs) through Precise St ructure Design Scientific Reports, Manuscript SREP-14-03662B, accepted

[19] J. Ran, L. Wu, T.W. Xu, Enhancement of Hydroxide conduction by self-assembly in Anion Conductive Comb-shaped copolymers, Polymer Chemistry, 2013, 4 (17), $4612-4620$.

[20]J. Pan, Y. Li, L. Zhuang, J.T. Lu, Self-crosslinked alkaline polymer electrolyte exceptionally stable at $90^{\circ} \mathrm{C}$, Chem. Commun., 2010, 46, 8597-8599.

[21]S. Gu, R. Cai, Y.S. Yan, Self-crosslinking for dimensionally stable and solvent-resistant quaternary phosphonium based hydroxide exchange membranes, Chem. Commun., 2011, 47, 2856-2858.

[22]N. J. Robertson, T. J. Clark, P. F. Mutolo, H. D. Abruna, G. W. Coates, Tunable High Performance Cross-Linked Alkaline Anion Exchange Membranes for Fuel Cell Applications, J. AM. CHEM. SOC. 2010, 132, 3400-3404.

[23] N.W. Li, L.Z. Wang and M. Hickner, Cross-linked comb-shaped anion exchange membranes with high base stability, Chem. Commun., 2014, 50, 4092-4095.

[24]L.Z. Wang and M. A. Hickner, Low-temperature crosslinking of anion exchange Membranes, Polym. Chem., 2014, 5, 2928-2935.

[25]Y. Sone, P. Ekdunge, D. Simonsson, Proton Conductivity of Nafion 117 as Measured by a Four - Electrode AC Impedance Method. Journal of the Electrochemical Society, 1996, 143, 1254-1259.

[26] V. Tricoli, Proton and methanol transport in poly (perfluorosulfonate) membranes containing $\mathrm{Cs}+$ and $\mathrm{H}+$ cations. Journal of the electrochemical society, 1998, 145, 3798-3801.

[27]J, R Varcoe, R.C.T. Slade, G.L. Wright, Y. Chen, Steady-state d.c. and impedance investigations of $\mathrm{H} 2 / \mathrm{O} 2$ alkaline membrane fuel cells with commercial Pt/C, Ag/C and Au/C cathodes. J Phys Chem B 2006, 110, 21041e9.

[28]J, R Varcoe, R.C.T. Slade, S.D. Poynton, D.J. Driscoll, D.C. Apperley, An 
ETFE-derived radiation-grafted anion-exchange membrane with properties specifically tailored for application in metal-cation-free alkaline polymer electrolyte fuel cells. Chem Mater 2007, 19, 2686e93.

[29] N. Li, T. Yan, Z. Li, T. Thurn-Albrecht and W. H. Binder, Energy Environ. Sci., 2012, 5, 7888-7892.

[30] S. Chempath, B. R. Einsla, L. R. Pratt, C. S., Macomber, J. M., Boncella, J. A. Rau, B.S. Pivovar, Density Functional Theory Study of Degradation of Tetraalkylammonium Hydroxides , J. Phys. Chem. C 2008, 112, 3179- 3182. 